\title{
Probing the Gluon Sivers Function with an Unpolarized Target: GTMD Distributions and the Odderons
}

\author{
Renaud Boussarie, ${ }^{1}$ Yoshitaka Hatta $\odot,{ }^{1}$ Lech Szymanowski®${ }^{2}$ and Samuel Wallon ${ }^{3,4}$ \\ ${ }^{1}$ Physics Department, Brookhaven National Laboratory, Upton, New York 11973, USA \\ ${ }^{2}$ National Centre for Nuclear Research (NCBJ), Pasteura 7, 02-093 Warsaw, Poland \\ ${ }^{3}$ Université Paris-Saclay, CNRS/IN2P3, IJCLab, 91405 Orsay, France \\ ${ }^{4}$ Sorbonne Université, Faculté de Physique, 4 place Jussieu, 75252 Paris Cedex 05, France
}

(Received 4 February 2020; revised manuscript received 26 March 2020; accepted 14 April 2020; published 29 April 2020)

\begin{abstract}
It is commonly believed that the Sivers function has uniquely to do with processes involving a transversely polarized nucleon. In this Letter we show that this is not necessarily the case. We demonstrate that exclusive pion production in unpolarized electron-proton scattering in the forward region is a direct probe of the gluon Sivers function due to its connection to the QCD odderon.
\end{abstract}

DOI: 10.1103/PhysRevLett.124.172501

Introduction.- Recently, there has been renewed interest in the interplay between spin physics and small- $x$ physics, largely motivated by the necessity to understand the small- $x$ behavior of the helicity distributions inside a longitudinally polarized proton. Generally speaking, polarization effects are suppressed by an inverse power of energy compared to unpolarized ones, but they are enhanced by double logarithms in energy. The resummation of these logarithms is a particularly challenging problem which has been first addressed by Bartels, Ermolaev, and Ryskin [1]. Recent activities include calculations of various higher order corrections [2] and the generalization to the orbital angular momentum sector [3], as well as alternative approaches to resummation [4-6].

Meanwhile, another very interesting, and rather unexpected interplay between spin and small- $x$ physics has been elucidated by Zhou [7] (see also Refs. [8-10]) in the transverse polarization sector. It was observed that the gluon Sivers function is related to the odderon in small- $x$ processes that are sensitive to transverse polarizations. The Sivers function [11] is a transverse momentum dependent (TMD) parton distribution function that has been exclusively discussed in the context of transverse single spin asymmetry (SSA). It turns out that the gluonic version of the Sivers function, despite its obvious connection to spin is not power suppressed by energy, but instead evolves with leading single (not double)

Published by the American Physical Society under the terms of the Creative Commons Attribution 4.0 International license. Further distribution of this work must maintain attribution to the author(s) and the published article's title, journal citation, and DOI. Funded by SCOAP . logarithms at small $x$. Moreover, this evolution equation is identical to that for the odderon amplitude in QCD which has been well established in the literature [12-15]. The numerical solution of the evolution equation [16] carries various phenomenological implications that can be tested in future measurements of SSA involving heavy quarks.

In this Letter, we dig into this correspondence to a deeper level and establish relations between the odderon and the so-called generalized TMDs (GTMDs) [17]. A similar study has been done in Ref. [18], but the target polarization effect was neglected. We show that, for spin-1/2 hadrons such as the proton, there are three independent GTMDs that can be associated with the odderon. Only one of them survives in the TMD (forward) limit and becomes the gluon Sivers function. Moreover, we observe that, once reinterpreted as a GTMD, the Sivers function is not necessarily tied to the transverse polarization of the incoming state. This opens up an intriguing possibility that one can experimentally access the gluon Sivers function in unpolarized collisions. Specifically, we calculate the differential cross section $d \sigma / d t$ for the exclusive process $e p \rightarrow e^{\prime} \gamma^{*} p \rightarrow e^{\prime} \pi^{0} p^{\prime}$, where both the incoming electron and proton are unpolarized. Since the (virtual) photon and the pion have opposite $C$ parities, at high energy this process is dominated by the odderon exchange which is $C$ odd. We then show that, among the three odderon GTMDs that contribute to this process, the one corresponding to the gluon Sivers function gives the leading contribution in the forward region $t \approx 0$.

Odderon and GTMDs. - In this section, we clarify the relation between the odderon amplitude and gluon GTMD distributions. The general parametrization of dipole-type gluon GTMDs can be easily adapted from the quark case [17] and reads [19,20] 


$$
\begin{aligned}
& \frac{4 M}{\bar{P}^{+}} \int \frac{d^{4} v}{(2 \pi)^{3}} \delta\left(v^{+}\right) e^{i x \bar{P}^{+} v^{-}-i(\boldsymbol{k} \cdot \boldsymbol{v})} \\
& \times\left\langle P^{\prime} S^{\prime}\left|\operatorname{Tr}\left[F^{i+}\left(-\frac{v}{2}\right) \mathcal{U}_{(v / 2),-(v / 2)}^{[+]} F^{i+}\left(\frac{v}{2}\right) \mathcal{U}_{-(v / 2),(v / 2)}^{[-]}\right]\right| P S\right\rangle \\
& =\bar{u}_{P^{\prime}}\left[F_{1,1}^{g}+i \frac{\sigma^{i+}}{\bar{P}^{+}}\left(\boldsymbol{k}^{i} F_{1,2}^{g}+\boldsymbol{\Delta}^{i} F_{1,3}^{g}\right)+i \frac{\sigma^{i j} \boldsymbol{k}^{i} \Delta^{j}}{M^{2}} F_{1,4}^{g}\right] u_{P},
\end{aligned}
$$

where the trace is in the fundamental representation and we defined the staple gauge links

$$
\mathcal{U}_{x, y}^{[ \pm]}=\left[x^{-}, \pm \infty\right]_{x}[\boldsymbol{x}, \boldsymbol{y}]_{ \pm \infty}\left[ \pm \infty, y^{-}\right]_{\boldsymbol{y}} .
$$

The notation $[x, y]_{z}$ denotes a straight Wilson line from $y$ to $x$ at fixed $z$. We defined $\bar{P}^{\mu}=\left[\left(P^{\mu}+P^{\prime \mu}\right) / 2\right], \Delta^{\mu}=P^{\prime \mu}-$ $P^{\mu}$ is the momentum transfer, and $M$ is the proton mass. The spinors with momenta $P$ and $P^{\prime}$ have associated spin vectors $S$ and $S^{\prime}$, which we will write explicitly hereafter. Boldface letters denote transverse vectors and $i, j=1,2$ are their indices. The four complex-valued GTMDs $F_{1, n}^{g}$ $(n=1,2,3,4)$ are functions of $(x, \xi, k, \Delta)$, where $\xi=$ $-\left(\Delta^{+} / 2 \bar{P}^{+}\right)$is the skewness parameter. The following symmetry properties are known [17]

$F_{1, n}^{g *}\left(x, \xi, \boldsymbol{k}^{2}, \boldsymbol{k} \cdot \boldsymbol{\Delta}, \boldsymbol{\Delta}^{2}\right)= \pm F_{1, n}^{g}\left(x,-\xi, \boldsymbol{k}^{2},-\boldsymbol{k} \cdot \boldsymbol{\Delta}, \boldsymbol{\Delta}^{2}\right)$,

with a $+\operatorname{sign}$ for $n=1,3,4$ and a $-\operatorname{sign}$ for $n=2$. In the following, we shall always be interested in the $\xi \simeq 0$ limit which is typically satisfied in small- $x$ kinematics. We can then write

$$
F_{1, n}^{g}=f_{1, n}+i \frac{(\boldsymbol{k} \cdot \boldsymbol{\Delta})}{M^{2}} g_{1, n},
$$

for $n=1,3,4$, and

$$
F_{1,2}^{g}=\frac{(\boldsymbol{k} \cdot \boldsymbol{\Delta})}{M^{2}} f_{1,2}+i g_{1,2},
$$

where $f$ and $g$ functions are real functions which depend on $x, \boldsymbol{k}^{2},|\boldsymbol{k} \cdot \boldsymbol{\Delta}|, \boldsymbol{\Delta}^{2}$. In the small $x$ approximation, we will set $x \simeq 0$ in the phase of the left-hand side (1.h.s.) of Eq. (1). Following the same manipulations as in Ref. [21], we can rewrite Eq. (1) as

$$
\begin{gathered}
\int d^{2} \boldsymbol{v} e^{-i(\boldsymbol{k} \cdot \boldsymbol{v})}\left\langle P^{\prime} S^{\prime}\left|\frac{1}{N_{c}} \operatorname{Tr}\left[U_{(\boldsymbol{v} / 2)} U_{-(\boldsymbol{v} / 2)}^{\dagger}\right]-1\right| P S\right\rangle \\
=(2 \pi)^{4} \delta\left(P^{+}-P^{\prime+}\right) \frac{\bar{P}^{+}}{4 M} \frac{g_{s}^{2}}{N_{c}\left(\boldsymbol{k}^{2}-\frac{\boldsymbol{\Delta}^{2}}{4}\right)} \\
\times \bar{u}_{P^{\prime} S^{\prime}}\left[F_{1,1}^{g}+i \frac{\sigma^{i+}}{\bar{P}^{+}}\left(\boldsymbol{k}^{i} F_{1,2}^{g}+\boldsymbol{\Delta}^{i} F_{1,3}^{g}\right)\right] u_{P S},
\end{gathered}
$$

where $U_{v}=[\infty,-\infty]_{v}$. The dependence on $x$ on the 1.h.s. is hidden in the regularization of the lightlike Wilson line operators in the small- $x$ effective formalism [22-24]. Note that the $F_{1,4}^{g}$ term has been neglected in Eq. (6). Indeed in the eikonal approximation $e^{i x \bar{P}^{+} v^{-}} \approx 1$, this term vanishes due to its $P T$ symmetry [25]. Also note that this expression is finite despite the denominator, due to the remarkable simplicity of dipole-type GTMDs, which are all proportional to this denominator. See, for example, Ref. [21]. The real, $(v \leftrightarrow-v)$-even part of the dipole operator is identified with the Pomeron, while the odderon is the imaginary, $(\boldsymbol{v} \leftrightarrow-\boldsymbol{v})$-odd part [13],

$$
\frac{1}{N_{c}} \operatorname{Tr}\left(U_{(\boldsymbol{v} / 2)} U_{-(\boldsymbol{v} / 2)}^{\dagger}\right)-1=\mathcal{P}(\boldsymbol{v})+i \mathcal{O}(\boldsymbol{v}) .
$$

It is easy to see that, on the 1.h.s. of Eq. (6), the Pomeron term is even in $\boldsymbol{k} \leftrightarrow-\boldsymbol{k}$, while the odderon term is odd under this exchange. The latter reads, with the parametrizations from Eqs. (4), (5),

$$
\begin{aligned}
\int d^{2} \boldsymbol{v} e^{-i(\boldsymbol{k} \cdot \boldsymbol{v})}\left\langle P^{\prime}, S^{\prime}|i \mathcal{O}(\boldsymbol{v})| P, S\right\rangle \\
=g_{s}^{2}(2 \pi)^{4} \delta\left(P^{\prime+}-P^{+}\right) \frac{\bar{P}^{+}}{4 M} \frac{\boldsymbol{k}^{j}}{N_{c}\left(\boldsymbol{k}^{2}-\frac{\boldsymbol{\Delta}^{2}}{4}\right)} \\
\quad \times \bar{u}_{P^{\prime}, S^{\prime}}\left[i \frac{\Delta^{j}}{M^{2}} g_{1,1}-\frac{\sigma^{i+}}{\bar{P}^{+}}\left(\delta^{i j} g_{1,2}+\frac{\Delta^{i} \Delta^{j}}{M^{2}} g_{1,3}\right)\right] u_{P, S} .
\end{aligned}
$$

This is the most general parametrization of the dipole-type odderon coupling to a generic spin- $1 / 2$ hadron. We see that the odderon is characterized by three independent GTMDs $g_{1, n}(n=1,2,3)$. It is interesting to notice that by using the Gordon identity in the first term

$$
\bar{u}_{P^{\prime} S^{\prime}} u_{P S}=\frac{M}{\bar{P}^{+}} \bar{u}_{P^{\prime} S^{\prime}} \gamma^{+} u_{P S}+\bar{u}_{P^{\prime} S^{\prime}} i \sigma^{+i} \frac{\Delta^{i}}{2 \bar{P}^{+}} u_{P S},
$$

we recognize a vector coupling between the odderon and the hadron, or "vector odderon" [26]. For the odderon to be a pure vector odderon, we would need the relations $g_{1,1}=2 g_{1,3}, g_{1,2}=0$. However, in general they are independent distributions. We also see that the vector odderon vanishes in the forward limit $\Delta \rightarrow 0$. In the near-forward, spin nonflip scattering of a proton, the three terms in Eq. (8) behave like

$$
i(\boldsymbol{k} \cdot \boldsymbol{\Delta}) g_{1,1}, \quad(\boldsymbol{k} \times \boldsymbol{s})_{z} g_{1,2}, \quad(\boldsymbol{k} \cdot \boldsymbol{\Delta})(\boldsymbol{\Delta} \times \boldsymbol{S})_{z} g_{1,3} .
$$

Since the odderon amplitude is a scalar function and is odd under $\boldsymbol{k} \rightarrow-\boldsymbol{k}$, it should be proportional to the vector $\boldsymbol{k}$, contracted by another two-dimensional vector. Equation (10) exhausts all possibilities in this kinematics. $g_{1,1}$, to which we will refer as the vector odderon term, is associated with momentum transfer $\boldsymbol{\Delta}$ (or impact parameter 
$\boldsymbol{b}$ in the coordinate space). This exists even for spinless hadrons, and the odderon coupling discussed in most literature $[7,12,18,27-30]$ is of this type. $g_{1,2}$ is the spindependent odderon [7]. To our knowledge, the structure of the last term $g_{1,3}$, let us call it the spin-vector odderon, has not been identified in the literature. This features a sine correlation between the azimuthal angles of $\boldsymbol{\Delta}$ and $\boldsymbol{S}$ which may lead to distinct experimental signals.

In the forward $\boldsymbol{\Delta}=\mathbf{0}$ limit, only $g_{1,2}=\operatorname{Im}\left(F_{1,2}^{g}\right)$ survives. If we further assume that the proton is transversely polarized, we find the familiar structure of the Sivers function

$\boldsymbol{k}^{2} \int d^{2} \boldsymbol{v} e^{-i(\boldsymbol{k} \cdot \boldsymbol{v})}\langle P S|i \mathcal{O}(\boldsymbol{v})| P S\rangle \propto 2 \frac{(\boldsymbol{k} \times \boldsymbol{S})_{z}}{M^{2}} \operatorname{Im}\left(F_{1,2}^{g}\right)$,

with the identification

$$
2 \operatorname{Im}\left(F_{1,2}^{g}\right)(x, 0, \boldsymbol{k}, \mathbf{0})=-x f_{1 T}^{\perp g}\left(x, \boldsymbol{k}^{2}\right) .
$$

Interestingly, and importantly for our purpose, the relevant spinor product is nonvanishing also for a generic helicity flip contribution

$$
\boldsymbol{k}^{i} \bar{u}_{P S^{\prime}} \sigma^{+i} u_{P S} \propto(\boldsymbol{k} \times \boldsymbol{h})_{z} \delta_{h,-h^{\prime}},
$$

where $\boldsymbol{h}=(1 / \sqrt{2})(1, i h)$ is a transverse vector built from proton helicity $h= \pm 1$ which resembles a circular polarization vector in the transverse plane. We thus find that the gluon Sivers function appears not only in processes involving a transversely polarized proton, but also in unpolarized processes where the proton spin flips. This includes unpolarized collisions where one independently sums over the polarizations of the initial and final proton helicities. This is the key observation that will be further explored in the next sections.

Exclusive diffractive pion production ep $\rightarrow e^{\prime} \pi^{0} p^{\prime}$ at small $x$.- In this section, we compute the differential cross section of exclusive pion production $e p \rightarrow e^{\prime} \pi^{0} p^{\prime}$ at high energy. The process is depicted in Fig. 1. Since the virtual photon and the pion have negative and positive $C$ parity, respectively, the exchanged object in the $t$ channel has negative $C$ parity. The process is thus a classic example of possible odderon signatures [31]. Instead of $\pi^{0}$, one can also consider $\eta_{c}$ [27]. However, the rate will be smaller and $\eta_{c}$ is more difficult to reconstruct. We will use the standard variables for deeply virtual meson production (DVMP)

$Q^{2}=-q^{2}, \quad x_{B}=\frac{Q^{2}}{2(P \cdot q)}, \quad t=\Delta^{2}, \quad y=\frac{(P \cdot q)}{(P \cdot \ell)}$.

Note that in the eikonal approximation, $t \approx-\Delta^{2}$. The value of skewness $\xi$ is approximately equal to $x \approx x_{B} / 2$ in this kinematics, and it is understood that all the GTMDs below are evaluated at $\xi \approx x$. Strictly speaking, this is different

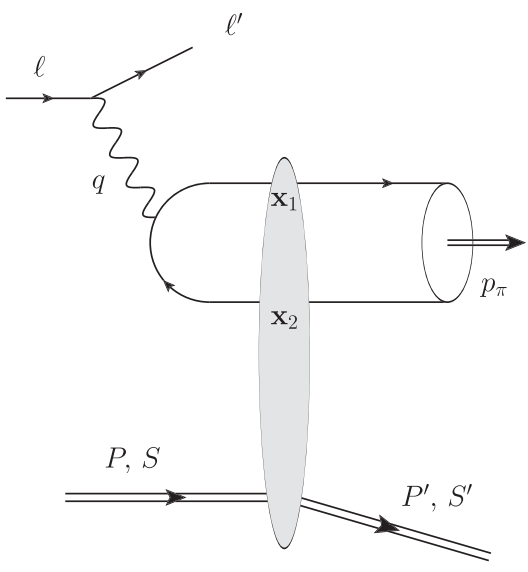

FIG. 1. Electroproduction of a $\pi^{0}$ meson at small $x$. The gray blob represents the interaction with the background field, and the white blob represents the distribution amplitude of the meson.

from the limit considered in the previous section $\xi=0$ at small but nonzero $x$. While the two limits are not the same in general, the small- $x$ evolution does not differentiate them at least in the leading logarithmic approximation. We thus identify the two limits but the difference should be kept in mind and scrutinized further. The computation of the amplitude at small $x$ performed in this Letter relies on the covariant background field methods [22-24,32-34]. The outgoing quark and antiquark fields in the shock wave background created by the target gluon fields are

$$
\begin{aligned}
\bar{\psi}_{\mathrm{eff}}\left(x_{0}\right)= & \theta\left(x_{0}^{-}\right) \bar{\psi}\left(x_{0}\right)+\theta\left(-x_{0}^{-}\right) \\
& \times \int d^{4} x_{1} \delta\left(x_{1}^{-}\right) \bar{\psi}\left(x_{1}\right) \gamma^{-} G\left(x_{10}\right)\left(U_{x_{1}}-1\right)
\end{aligned}
$$

for the quark, and

$$
\begin{aligned}
\psi_{\mathrm{eff}}\left(x_{0}\right)= & \theta\left(x_{0}^{-}\right) \psi\left(x_{0}\right)-\theta\left(-x_{0}^{-}\right) \\
& \times \int d^{4} x_{2} \delta\left(x_{2}^{-}\right) G\left(x_{02}\right) \gamma^{-} \psi\left(x_{2}\right)\left(U_{x_{2}}^{\dagger}-1\right)
\end{aligned}
$$

for the antiquark, where $x_{1,2}^{-}=0$ is the time of interaction with the shockwave and $G$ is the free fermion propagator. From these two effective fields, we can build the amplitude for DVMP as

$$
\begin{aligned}
\mathcal{A}= & \frac{e_{f} e_{\ell}}{4 N_{c}} \bar{u}_{\ell^{\prime}} \gamma^{\mu} u_{\ell} \int d^{4} x_{0} d^{4} x_{1} d^{4} x_{2} \theta\left(-x_{0}^{-}\right) \delta\left(x_{1}^{-}\right) \delta\left(x_{2}^{-}\right) \\
& \times\left\langle\pi^{0}\left|\bar{\psi}\left(x_{1}\right) \Gamma^{\lambda} \psi\left(x_{2}\right)\right| 0\right\rangle\left\langle P^{\prime}\left|\operatorname{Tr}\left(U_{x_{1}} U_{x_{2}}^{\dagger}\right)-N_{c}\right| P\right\rangle \\
& \times e^{-i\left(q \cdot x_{0}\right)} G_{\mu \nu}(q) \operatorname{Tr}\left[\gamma^{-} G\left(x_{10}\right) \gamma^{\nu} G\left(x_{02}\right) \gamma^{-} \Gamma_{\lambda}\right],
\end{aligned}
$$

where $e_{f}$ is an effective electric charge which takes into account the flavor content of the meson, and $G_{\mu \nu}$ is the photon propagator and the sum over Fierz matrices $\Gamma_{\lambda}$ is implied. We evaluate this at leading $s$-channel twist by 
taking the light cone expansion of the bilocal vacuum-tomeson correlator $\left\langle\pi^{0}|\bar{\psi} \psi| 0\right\rangle$. Projecting onto the axialvector state $\left(\Gamma^{\lambda}\right)\left(\Gamma_{\lambda}\right) \rightarrow\left(\gamma^{\lambda} \gamma_{5}\right)\left(\gamma_{5} \gamma_{\lambda}\right)$ to take into account the properties of the leading twist chiral-even DA for the pion

$$
\begin{aligned}
& \left\langle\pi\left(p_{\pi}\right)\left|\bar{\psi}\left(x_{1}\right) \gamma^{\lambda} \gamma_{5} \psi\left(x_{2}\right)\right| 0\right\rangle \\
& \quad=i f_{\pi} p_{\pi}^{\lambda} \int_{0}^{1} d z e^{i z\left(p_{\pi} \cdot x_{1}\right)+i \bar{z}\left(p_{\pi} \cdot x_{2}\right)} \phi_{\pi}(z),
\end{aligned}
$$

and following computation steps are very similar to what is described in Ref. [35]; one gets

$$
\begin{aligned}
\mathcal{A}= & \frac{-e_{f} e_{\ell} f_{\pi}}{N_{c}} \delta\left(q^{-}-p_{\pi}^{-}\right) \bar{u}_{\ell^{\prime}} \gamma^{\mu} u_{\ell} \int d^{2} \boldsymbol{b} e^{i\left(\boldsymbol{q}-\boldsymbol{p}_{\pi}\right) \cdot \boldsymbol{b}} \\
& \times \epsilon^{\alpha \beta+-} \int d^{2} \boldsymbol{r} \int_{0}^{1} d z \phi_{\pi}(z) r_{\perp \alpha} \sqrt{\frac{z \bar{z} Q^{2}}{\boldsymbol{r}^{2}}} K_{1}\left(\sqrt{z \bar{z} Q^{2} \boldsymbol{r}^{2}}\right) \\
& \times\left\langle P^{\prime}\left|\operatorname{Tr}\left(U_{\boldsymbol{b}+\bar{z} \boldsymbol{r}} U_{\boldsymbol{b}-z \boldsymbol{r}}^{\dagger}-1\right)\right| P\right\rangle G_{\mu \nu}(q)\left(q^{-} g_{\perp \beta}^{\nu}-n^{\nu} q_{\perp \beta}\right),
\end{aligned}
$$

where $\bar{z}=1-z \cdot \phi_{\pi}$ is the pion's leading twist axial-vector distribution amplitude (DA) and $f_{\pi}=131 \mathrm{MeV}$ is the decay constant. As expected, the amplitude (19) involves the $t$ channel exchange of an odderon. Indeed, the symmetry property of the DA $\phi_{\pi}(z)=\phi_{\pi}(\bar{z})$ means that the $\boldsymbol{r} \leftrightarrow-\boldsymbol{r}$ antisymmetric contribution from the dipole matrix element contributes, i.e., its imaginary part is selected by the $C$ parity of the process. To make the connection to the odderon GTMDs explicit, we use the Fourier transform of Eq. (8), which is a particularly simple case of the general formula derived in Ref. [36]. Using these results, Eq. (19) then becomes

$$
\begin{aligned}
\mathcal{A}= & \frac{e_{f} e_{\ell} f_{\pi}}{N_{c}} g_{s}^{2}(2 \pi)^{5} \delta\left(p_{\pi}+\Delta-q\right) \bar{u}_{\ell^{\prime}} \gamma^{\mu} u_{\ell} \\
& \times G_{\mu \nu}(q) \epsilon^{\alpha \beta+-}\left(q^{-} g_{\perp \beta}^{\nu}-n^{\nu} q_{\perp \beta}\right) \int_{0}^{1} d z \phi_{\pi}(z) \\
& \times \frac{\bar{P}^{+}}{4 M} \int \frac{d^{2} \boldsymbol{k}}{\boldsymbol{k}^{2}-\frac{\boldsymbol{\Delta}^{2}}{4}} \frac{\boldsymbol{k}^{j}\left(k_{\perp \alpha}+\frac{z-\bar{z}}{2} \Delta_{\perp \alpha}\right)}{\left(\boldsymbol{k}+\frac{z-\bar{z}}{2} \Delta\right)^{2}+z \bar{z} Q^{2}} \\
& \times \bar{u}_{P^{\prime}, S^{\prime}}\left[i \frac{\Delta^{j}}{M^{2}} g_{1,1}-\frac{\sigma^{i+}}{\bar{P}^{+}}\left(\delta^{i j} g_{1,2}+\frac{\boldsymbol{\Delta}^{i} \Delta^{j}}{M^{2}} g_{1,3}\right)\right] u_{P, S} .
\end{aligned}
$$

Let us focus on the small $\boldsymbol{\Delta}$ limit in which Eq. (20) simplifies drastically. Only $g_{1,2} \propto f_{1 T}^{\perp g}$ survives in this limit and the amplitude becomes proportional to

$$
\begin{aligned}
\mathcal{A} & \propto \int \frac{d^{2} \boldsymbol{k}}{\boldsymbol{k}^{2}+z \bar{z} Q^{2}} x f_{1 T}^{\perp g}\left(x, \boldsymbol{k}^{2}\right) \\
& =-\frac{1}{z \bar{z} Q^{2}} \int \frac{d^{2} \boldsymbol{k} \boldsymbol{k}^{2}}{\boldsymbol{k}^{2}+z \bar{z} Q^{2}} x f_{1 T}^{\perp g}\left(x, \boldsymbol{k}^{2}\right),
\end{aligned}
$$

where in the second line we used the relation

$$
\int d^{2} \boldsymbol{k x} f_{1 T}^{\perp g}\left(x, \boldsymbol{k}^{2}\right)=0 .
$$

This relation was first noted in Ref. [7] within a specific model, but it is actually a general result [37].

In order to get the cross section, one has to square Eq. (20) and sum over the initial and final proton spins. As can be seen from Eq. (13), a nonvanishing contribution arises when the proton flips spin $S=-S^{\prime}$. Taking into account the quark content of the pion $\left|\pi^{0}\right\rangle=(|u \bar{u}\rangle-$ $|d \bar{d}\rangle) / \sqrt{2}$ so that $e_{f}=\left(e_{u}-e_{d}\right) / \sqrt{2}=e / \sqrt{2}$, we thus arrive at the main result of this Letter

$$
\begin{aligned}
& \frac{d \sigma}{d x_{B} d Q^{2} d|t|}=\frac{\pi^{5} \alpha_{\mathrm{em}}^{2} \alpha_{s}^{2} f_{\pi}^{2}}{2^{4} x_{B} N_{c}^{2} M^{2} Q^{6}}\left(1-y+\frac{y^{2}}{2}\right) \\
& \times\left[\int_{0}^{1} d z \frac{\phi_{\pi}(z)}{z \bar{z}} \int d \boldsymbol{k}^{2} \frac{\boldsymbol{k}^{2}}{\boldsymbol{k}^{2}+z \bar{z} Q^{2}} x f_{1 T}^{\perp g}\left(x, \boldsymbol{k}^{2}\right)\right]^{2},
\end{aligned}
$$

valid in the forward region $t \approx 0$, and for perturbative values of $Q$. The corrections to this formula are of order $t / M^{2}$, where $M$ is the target mass. Quite remarkably, the leading contribution to this unpolarized observable is given by the gluon Sivers function which is usually associated with a transversely polarized nucleon, and this has been missed in all the previous calculations. However, some moderate $x$ models suggested that this reaction can probe the nucleon tensor charge [38]. We expect that a similar conclusion holds in related observables such as $\eta_{c}$ production. It is easy to check by retracing the steps from Eqs. (20)-(23) that only the transversely polarized virtual photon contributes to this formula. In the large- $Q^{2}$ region, the cross section is directly related to the $C$-odd collinear three-gluon correlator $O\left(x_{1}, x_{2}\right)$ relevant to single spin asymmetry $[7,39]$

$$
\int d \boldsymbol{k}^{2} \boldsymbol{k}^{2} x f_{1 T}^{\perp g}\left(x, \boldsymbol{k}^{2}\right) \propto O(x, x)+O(x, 0) .
$$

Note, however, that neglecting $\boldsymbol{k}^{2}$ in the denominator of Eq. (23) results in an end-point singularity at $z, \bar{z}=0$. In practice, this should be cutoff at $z, \bar{z} \sim \Lambda^{2} / Q^{2}$, leading to a logarithmic enhancement $\ln ^{2} Q^{2} / \Lambda^{2}$ at large $Q^{2}$. As an example, consider the asymptotic form $\phi_{\pi}(z)=6 z \bar{z}$ and a simple model for the Sivers function at small $x$ built in Ref. [7],

$$
x f_{1 T}^{\perp g}\left(x, \boldsymbol{k}^{2}\right)=\mathcal{C} \frac{\boldsymbol{k}^{2}}{\Lambda^{4}}\left(2-\frac{\boldsymbol{k}^{2}}{\Lambda^{2}}\right) e^{-\boldsymbol{k}^{2} / \Lambda^{2}},
$$

where $\mathcal{C}$ is a dimensionless constant. We find 


$$
\begin{aligned}
& \frac{d \sigma}{d x_{B} d Q^{2} d|t|}=\pi^{5} \frac{\alpha_{\mathrm{em}}^{2} \alpha_{s}^{2} f_{\pi}^{2} Q^{2}}{x_{B} M^{2}}\left(1-y+\frac{y^{2}}{2}\right) \\
& \times \frac{\mathcal{C}^{2}}{\Lambda^{8}}\left[\int d \alpha \frac{\alpha^{2}\left(2-\frac{Q^{2}}{\Lambda^{2}} \alpha\right)}{\sqrt{1+4 \alpha}} \ln \left(\frac{\sqrt{1+4 \alpha}+1}{\sqrt{1+4 \alpha}-1}\right) e^{-\alpha\left(Q^{2} / \Lambda^{2}\right)}\right]^{2} .
\end{aligned}
$$

For $\Lambda \ll Q$, the exponent peaks the integrand around $\alpha=0$, which allows for a fully analytical integration:

$$
\begin{aligned}
\frac{d \sigma}{d x_{B} d Q^{2} d|t|}= & \pi^{5} \frac{\alpha_{\mathrm{em}}^{2} \alpha_{s}^{2} f_{\pi}^{2}}{x_{B} M^{2}}\left(1-y+\frac{y^{2}}{2}\right) \\
& \times \mathcal{C}^{2} \frac{\Lambda^{4}}{Q^{10}}\left[5-2 \gamma_{E}+2 \log \left(\frac{\Lambda^{2}}{Q^{2}}\right)\right]^{2} .
\end{aligned}
$$

Discussions.-That the cross section $d \sigma / d t$ does not vanish in the forward limit $\Delta \rightarrow 0$ is quite nontrivial. As we mentioned above, only the transversely polarized virtual photon contributes to the cross section. The polarization vector of the photon must then be contracted with another transverse vector. For the case of pion production, and without considering proton spin effects, the only available vector is momentum transfer $\Delta$. This is why the odderon contribution to $d \sigma / d t$ vanishes in the $t \approx-\Delta^{2} \rightarrow 0$ limit in Refs. [27,30,40] (see also Ref. [41]). In contrast, Ref. [31] obtained a finite result in the same limit. This is because the authors of Ref. [31] considered processes where the proton is excited to a negative parity resonance $N^{*}$, which is modeled as a $p$-wave bound state of a diquark and a quark. The $p$-wave wave function involves a transverse vector which can be contracted with the photon polarization vector, thereby giving a finite result at $t=0$. We, however, note that considering the excitation and decay of the target proton introduces extra theoretical uncertainties.

Our central observation in this paper is that the required vector can come from the spin- $1 / 2$ nature of the proton. The spin-dependent odderon accompanies the spinor product $\bar{u} \sigma^{+i} u$, which carries a transverse vector index. This is nonvanishing provided the proton flips spin $S^{\prime}=-S$ [see Eq. (13)], and such spin-flip contributions are automatically included when calculating unpolarized cross sections. One may wonder why the proton can flip helicity in the eikonal approximation in the forward limit. Equation (8) shows that this can occur nonperturbatively, assisted by the transverse vector $\boldsymbol{k}$ which carries one unit of angular momentum. It is also a necessary consequence of the existence of the spindependent odderon.

We should add that our calculation is valid when $Q^{2}$ is perturbative, say, $Q^{2}>$ a few $\mathrm{GeV}^{2}$ since this is the only hard scale. Some might be tempted to assume that at sufficiently high energy, the gluon saturation scale $Q_{s}$ could serve as a hard scale. However, as demonstrated in Ref. [16], the characteristic momentum scale of the odderon amplitude does not scale with $Q_{s}$.
The previous odderon search at HERA [42] measured neutrons from the reaction $p \rightarrow N^{*} \rightarrow n$ based on a calculation in Ref. [31], and no signal was observed. We propose to measure the elastically scattered proton in the final state instead. In the near forward region, one should see the flattening of the curve $d \sigma / d t$ as $t \rightarrow 0$ before reaching the (diverging) contribution from the Primakoff process $\gamma^{*} \gamma \rightarrow \pi^{0}$ in the small- $t$ limit:

$$
\frac{d \sigma^{\text {Primakoff }}}{d x_{B} d Q^{2} d|t|} \approx \frac{(2 \pi) \alpha_{e m}^{4} f_{\pi}^{2}\left(1+(1-y)^{2}\right)}{x_{B} Q^{6}|t|}\left[\int_{0}^{1} d z \frac{\phi_{\pi}(z)}{\phi_{\pi}^{\infty}(z)}\right]^{2},
$$

where $\phi_{\pi}^{\infty}$ is the asymptotic DA $\phi_{\pi}^{\infty}(z)=6 z \bar{z}$, and the leading twist approximation was taken as a first estimate. Despite the relative suppression factor $\alpha_{e m}^{2}$, this can be a serious background at small $t$ and large $Q^{2}$. Fortunately, there is no interference between the spin-dependent odderon and the (leading) Primakoff amplitude because the proton helicity flips in the former but not in the latter. Therefore in principle one can subtract Eq. (28) from the measured cross section. In practice some subleading corrections to Eq. (28) or to the forward limit could complicate this task. For larger values of $t$, the Primakoff process should be negligible and one can probe the three types of odderon GTMDs $g_{1,1}, g_{1,2}$, and $g_{1,3}$. However, the cross section formula in this region is rather complicated.

Conclusions.-In this Letter, we have shown that GTMDs provide a unified framework to treat the spindependent and spin-independent Odderons on an equal footing. The formula (8), which involves three independent dipole gluon GTMDs, describes how the odderon couples to generic spin- $1 / 2$ hadrons. While discussions of odderonhadron coupling are scattered in the literature, it has not been previously presented in this most general, coherent form. Of particular interest is the spin-dependent odderon $g_{1,2}$ which reduces to the gluon Sivers function in the forward (TMD) limit [7]. We have demonstrated that this function gives the dominant contribution to exclusive pion production ep $\rightarrow e^{\prime} \pi^{0} p^{\prime}$ in the forward region $t \approx 0$.

It would be very interesting to test our prediction at existing and future ep colliders such as the EIC, and especially, the $\mathrm{LHeC}$ where the target polarization is not planned at the moment. The detection of events will be an unambiguous signal of the much sought-after QCD odderon, and at the same time, shed light on the magnitude of the gluon Sivers effect which remains largely mysterious to date.

A lot of progress has been made for one-loop corrections to exclusive diffractive processes at small $x$ [43-46]. In particular, a similar computation to that of Ref. [45], adapted from results of Ref. [44], would easily provide the one-loop corrections to the process described in this 
Letter, furthering the precision for $\mathrm{EIC}$ and $\mathrm{LHeC}$ predictions.

The authors are grateful to A. V. Grabovsky for his collaboration at the early stages of this work, and to B. Pire for insightful discussions. This work is supported by the U.S. Department of Energy, Office of Science, Office of Nuclear Physics, under contract No. DE- SC0012704, and in part by Laboratory Directed Research and Development (LDRD) funds from Brookhaven Science Associates. This project has received funding from the European Union's Horizon 2020 research and innovation program STRONG2020 under grant Agreement No. 824093. L. S. thanks the CNRS for support. The work of L.S. is also partially supported by Grant No. 2017/26/M/ST2/01074 of the National Science Center in Poland and by French-Polish Collaboration Agreement POLONIUM.

[1] J. Bartels, B. I. Ermolaev, and M. G. Ryskin, Z. Phys. C 72, 627 (1996).

[2] B. I. Ermolaev, M. Greco, and S. I. Troyan, Phys. Lett. B 579, 321 (2004).

[3] R. Boussarie, Y. Hatta, and F. Yuan, Phys. Lett. B 797, 134817 (2019).

[4] Y. V. Kovchegov, D. Pitonyak, and M. D. Sievert, J. High Energy Phys. 01 (2016) 072; 10 (2016) 148(E).

[5] Y. V. Kovchegov, J. High Energy Phys. 03 (2019) 174.

[6] F. Cougoulic and Y. V. Kovchegov, Phys. Rev. D 100, 114020 (2019).

[7] J. Zhou, Phys. Rev. D 89, 074050 (2014).

[8] L. Szymanowski and J. Zhou, Phys. Lett. B 760, 249 (2016).

[9] D. Boer, M. G. Echevarria, P. J. Mulders, and J. Zhou, Phys. Rev. Lett. 116, 122001 (2016).

[10] H. Dong, D.-X. Zheng, and J. Zhou, Phys. Lett. B 788, 401 (2019).

[11] D. W. Sivers, Phys. Rev. D 41, 83 (1990).

[12] Y. V. Kovchegov, L. Szymanowski, and S. Wallon, Phys. Lett. B 586, 267 (2004).

[13] Y. Hatta, E. Iancu, K. Itakura, and L. McLerran, Nucl. Phys. A760, 172 (2005).

[14] A. V. Grabovsky, J. High Energy Phys. 09 (2013) 141.

[15] R. E. Gerasimov and A. V. Grabovsky, J. High Energy Phys. 04 (2013) 102.

[16] X. Yao, Y. Hagiwara, and Y. Hatta, Phys. Lett. B 790, 361 (2019).

[17] S. Meissner, A. Metz, and M. Schlegel, J. High Energy Phys. 08 (2009) 056.

[18] D. Boer, T. Van Daal, P. J. Mulders, and E. Petreska, J. High Energy Phys. 07 (2018) 140.
[19] S. Bhattacharya, A. Metz, V. K. Ojha, J.-Y. Tsai, and J. Zhou, arXiv:1802.10550.

[20] C. Lorc and B. Pasquini, J. High Energy Phys. 09 (2013) 138.

[21] Y. Hatta, B.-W. Xiao, and F. Yuan, Phys. Rev. Lett. 116, 202301 (2016).

[22] I. Balitsky, Nucl. Phys. B463, 99 (1996).

[23] I. Balitsky, Phys. Rev. Lett. 81, 2024 (1998).

[24] I. Balitsky, Phys. Rev. D 60, 014020 (1999).

[25] Y. Hatta, Y. Nakagawa, B. Xiao, F. Yuan, and Y. Zhao, Phys. Rev. D 95, 114032 (2017).

[26] C. Ewerz, M. Maniatis, and O. Nachtmann, Ann. Phys. (Amsterdam) 342, 31 (2014).

[27] J. Czyzewski, J. Kwiecinski, L. Motyka, and M. Sadzikowski, Phys. Lett. B 398, 400 (1997); 411, 402(E) (1997).

[28] Y. V. Kovchegov and M. D. Sievert, Phys. Rev. D 86, 034028 (2012); 86, 079906(E) (2012).

[29] A. Dumitru, G. A. Miller, and R. Venugopalan, Phys. Rev. D 98, 094004 (2018).

[30] A. Dumitru and T. Stebel, Phys. Rev. D 99, 094038 (2019).

[31] E. R. Berger, A. Donnachie, H. G. Dosch, W. Kilian, O. Nachtmann, and M. Rueter, Eur. Phys. J. C 9, 491 (1999).

[32] L. D. McLerran and R. Venugopalan, Phys. Rev. D 49, 2233 (1994).

[33] L. D. McLerran and R. Venugopalan, Phys. Rev. D 49, 3352 (1994).

[34] L. D. McLerran and R. Venugopalan, Phys. Rev. D 50, 2225 (1994).

[35] R. Boussarie, Y. Hatta, B.-W. Xiao, and F. Yuan, Phys. Rev. D 98, 074015 (2018).

[36] T. Altinoluk and R. Boussarie, J. High Energy Phys. 10 (2019) 208.

[37] J. Zhou (private communication).

[38] S. Ahmad, G. R. Goldstein, and S. Liuti, Phys. Rev. D 79, 054014 (2009).

[39] H. Beppu, Y. Koike, K. Tanaka, and S. Yoshida, Phys. Rev. D 82, 054005 (2010).

[40] R. Engel, D. Yu. Ivanov, R. Kirschner, and L. Szymanowski, Eur. Phys. J. C 4, 93 (1998).

[41] L. A. Harland-Lang, V. A. Khoze, A. D. Martin, and M. G. Ryskin, Phys. Rev. D 99, 034011 (2019).

[42] C. Adloff et al. (H1 Collaboration), Phys. Lett. B 544, 35 (2002).

[43] R. Boussarie, A. V. Grabovsky, L. Szymanowski, and S. Wallon, J. High Energy Phys. 09 (2014) 026.

[44] R. Boussarie, A. V. Grabovsky, L. Szymanowski, and S. Wallon, J. High Energy Phys. 11 (2016) 149.

[45] R. Boussarie, A. V. Grabovsky, D. Y. Ivanov, L. Szymanowski, and S. Wallon, Phys. Rev. Lett. 119, 072002 (2017).

[46] R. Boussarie, A. V. Grabovsky, L. Szymanowski, and S. Wallon, Phys. Rev. D 100, 074020 (2019). 